\title{
Radiological and clinical features of primary NK/T-cell lymphoma involving the whole length of the esophagus: A case report
}

\author{
PAN LIANG, XIU-CHUN REN and JIAN-BO GAO \\ Department of Radiology, The First Affiliated Hospital, Zhengzhou University, Zhengzhou, Henan 450052, P.R. China
}

Received May 19, 2016; Accepted June 13, 2017

DOI: $10.3892 / 01.2017 .6456$

\begin{abstract}
Primary esophageal natural killer (NK)/T-cell lymphoma is a markedly rare tumor. There were only 6 cases of the disease identified prior to June 2015. In the present study, the aforementioned cases were validated, and relevant computed tomography $(\mathrm{CT})$ results and clinical features of primary esophageal NK/T-cell lymphoma were determined, to increase awareness of this type of tumor. CT features and clinical presentations of a patient with pathologically confirmed esophageal NK/T-cell lymphoma was analyzed. The patient exhibited non-specific clinical symptoms and CT images revealed diffuse thickening of the entire length of the esophagus. The patient received cycles of systemic chemotherapy and subsequent chest CT images demonstrated prompt and marked shrinkage of the tumor. At the time of writing, the patient has survived for 24 months and experiences a good quality of life without postprandial fullness or difficulty swallowing solid food. In addition, the characteristics of 6 patients with complete clinical features of this type of tumor, on the basis of a review of published studies (online PubMed, Medline, Google Scholar, Chinese Biomedicine Database and China Journal Full Text Database search), were retrospectively analyzed. Although primary esophageal NK/T-cell lymphoma is a markedly rare tumor, it is considered to be included in differential diagnosis of patients presenting with a fungal or viral infection, therapy-related mucositis or reflux esophagitis. The final diagnosis of primary esophageal NK/T-cell lymphoma is on the basis of a combination of clinical, CT and histopathological results.
\end{abstract}

Correspondence to: Dr Jian-Bo Gao, Department of Radiology, The First Affiliated Hospital, Zhengzhou University, 1 Eastern Jianshe Road, Zhengzhou, Henan 450052, P.R. China

E-mail: cjr.gaojianbo@vip.163.com

Abbreviations: NK/T-cell lymphoma, natural killer T cell lymphoma; NHL, non-Hodgkin's lymphoma; CT, computed tomography; EBV, Epstein-Barr virus

Key words: natural killer/T-cell lymphoma, esophagus, computed tomography, digestive system cancer, upper gastrointestinal tract

\section{Introduction}

Natural killer (NK)/T-cell lymphoma, an aggressive malignancy, is a unique type of non-Hodgkin's lymphoma (NHL) (1). It primarily occurs in the nasal/nasopharyngeal region, accounting for $75 \%$ of cases, with $4 \%$ occurring in the skin and $6 \%$ in the gastrointestinal tract (2). However, primary lymphoma located within the esophagus is markedly rare, with an incidence of $<1 \%$ of all patients with lymphoma (3) and the majority of such cases are NHL. Furthermore, to the best of our knowledge, only 6 cases of primary NK/T-cell lymphoma of the esophagus have been identified (4-8). Owing to the poorly defined early clinical and radiological signs, cases of primary esophageal NK/T-cell lymphoma present difficulties to physicians; therefore, appropriate preoperative diagnosis and operative planning may be delayed.

In the present study discussed the case report of a 52-year-old male patient with esophageal NK/T-cell lymphoma who presented with a 2-year history of postprandial fullness and difficulty swallowing solid food, and was treated accordingly. Primary esophageal NK/T-cell lymphoma is a markedly rare tumor and is considered during the differential diagnosis of patients presenting with a fungal or viral infection; however, only few studies have reported cases of this cancer type. In the present study, the imaging characteristics and clinical features of a rare case of primary esophageal NK/T-cell lymphoma were analyzed, in addition, the results of previous studies regarding this cancer were reviewed.

\section{Case report}

A 52-year-old male presented to the First Affiliated Hospital of Zhengzhou University (Zhengzhou, China) with a 2-year history of postprandial fullness and difficulty swallowing solid food. The patient did not complain of nausea, vomiting, fever, weight loss or night sweats, and the physical examination and laboratory blood tests were normal. In addition, the results of coagulation studies, electrolyte level tests and tests of renal and liver function were all within the normal limits. Increased levels of tumor markers including cancer antigen (CA)125, CA199 and carcinoembryonic antigen were not observed. A bone marrow biopsy was negative for lymphoma.

An esophagogastroduodenoscopy (Fig. 1) revealed diffuse mucous membrane congestion and edema located along the entire length of the esophagus. Additionally, multiple ovoid 

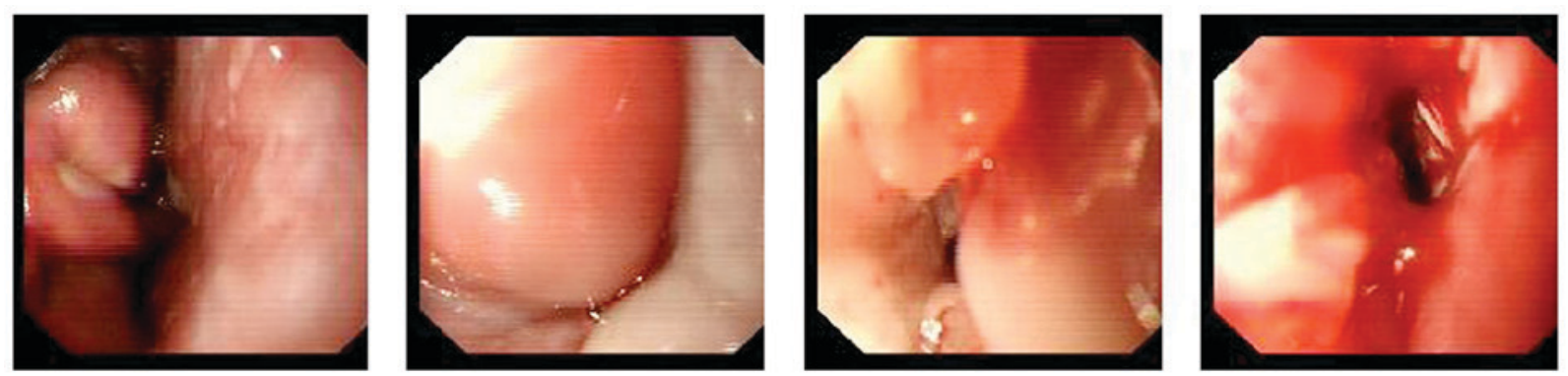

Figure 1. Esophagogastroduodenoscopy revealed multiple ovoid lesions with intact mucosa starting within $20 \mathrm{~cm}$ of the incisor teeth with diffuse mucous membrane congestion and edema located along the whole length of the esophagus.
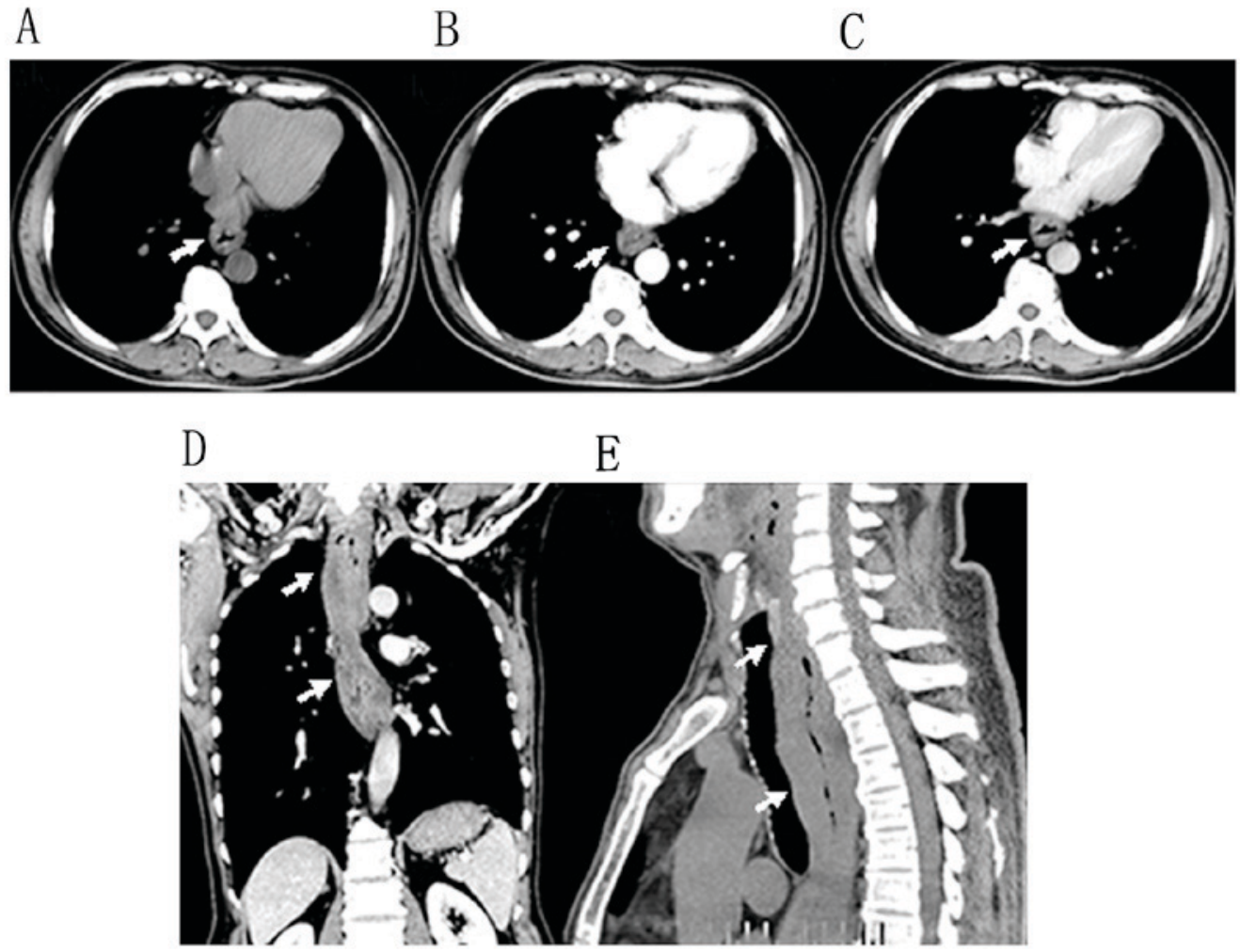

Figure 2. CT of the esophagus demonstrated diffuse thickening of the esophageal wall and a well-defined poorly enhanced mass without enlarged lymph nodes in the centrilobular and paraseptal regions (arrows). (A) Plain CT scan image; (B) arterial phase of contrast-enhanced CT image; (C) parenchymal phase of contrast-enhanced CT scan image; (D) arterial phase of contrast-enhanced CT scan image; and (E) plain CT scan image. CT, computed tomography.

lesions with intact normal mucosa were identified within $20 \mathrm{~cm}$ of the incisor teeth. The remaining esophagus was irregular and stenotic which prevented the esophagogastroduodenoscope from passing through. Biopsy specimens from six esophageal sites of endoscopic mucosal resection were obtained for pathological evaluation. A chest computed tomography (CT) scan (Fig. 2A-E) disclosed diffuse thickening of the esophageal wall, with apparent stenosis of the esophagus. A dynamic chest $\mathrm{CT}$ identified a well-defined poorly enhanced mass in the abnormal esophagus (Fig. 2B and C). No enlarged lymph nodes were detected in the centrilobular or paraseptal regions.

Histopathological examination of the biopsies of the esophagus revealed moderate pleomorphic atypical lymphoid cells disseminated in the lesions, with irregular nuclei and a pale cytoplasm (Fig. 3A). Immunohistochemistry was performed on formalin-fixed paraffin-embedded tissue sections using the streptavidin biotin peroxidase method (9). Immunohistochemical staining of the biopsies were positive for cluster of differentiation (CD)3 (Fig. 3B), CD56 (Fig. 3C) and Ki-67 (90\%; Fig. 3D); weakly positive for granzyme B (Fig. 3E) and cytotoxic granule-associated RNA-binding protein (TIA-1) (Fig. 3F). However, biopsies were negative for CD20 and cytokeratin. The results of the present study suggested the diagnosis of NK/T-cell lymphoma.

The patient was staged as IIA, according to the Ann Arbor system (7), and treated with 5 cycles of a cisplatine [50 mg day (d)1; $60 \mathrm{mg} \mathrm{d} 2 \mathrm{~d} 3$ ], etoposide VP-16 (180 mg d1-d3), ifosfamide ( $2 \mathrm{~g} \mathrm{~d} 1-\mathrm{d} 3)$, dexamethasone (40 $\mathrm{mg} \mathrm{d1}-\mathrm{d} 4)$ and mesna (0.4 g d1-d3) therapy regimen. Chest CT studies following chemotherapy revealed prompt and marked shrinkage of the tumor (Fig. 4). The patient remained in remission until the last 
A

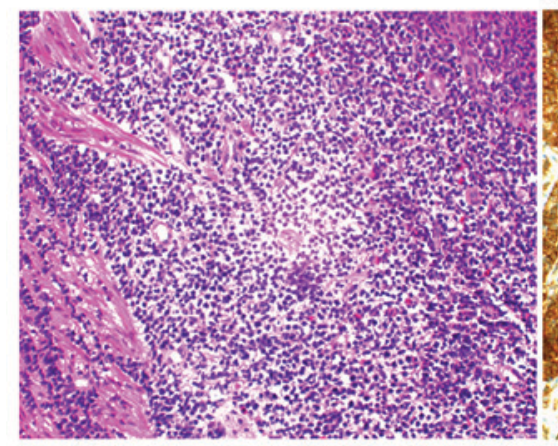

D

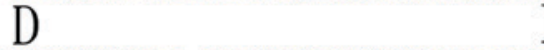

B

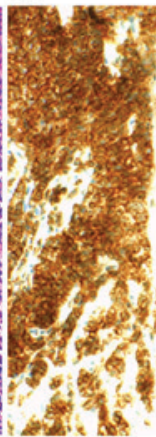

E
C

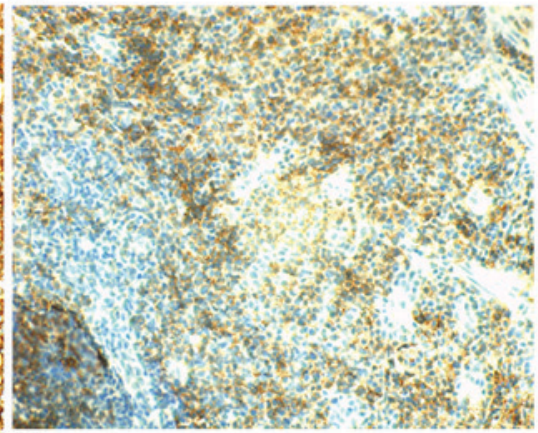

F

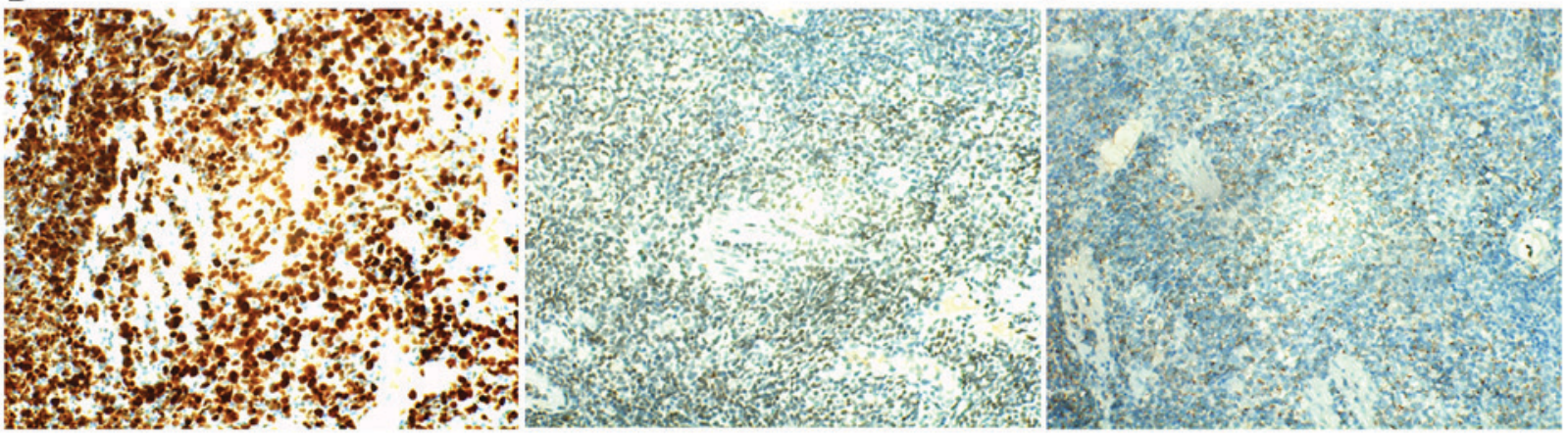

Figure 3. Histopathological examination of esophageal biopsy specimens revealed large numbers of moderately sized pleomorphic atypical lymphoid cells disseminated in the lesions. Immunohistochemical staining was positive for CD3, CD56 and Ki-67 (90\%); weakly positive for granzyme B and TIA-1; and negative for CD20 and cytokeratin. Staining for (A) hematoxylin and eosin; (B) CD3; (C) CD56; (D) Ki-67; (E) granzyme B; and (F) TIA-1. Magnification, $\mathrm{x}$ 400. CD, cluster of differentiation; TIA-1, TIA1 cytotoxic granule-associated RNA-binding protein.
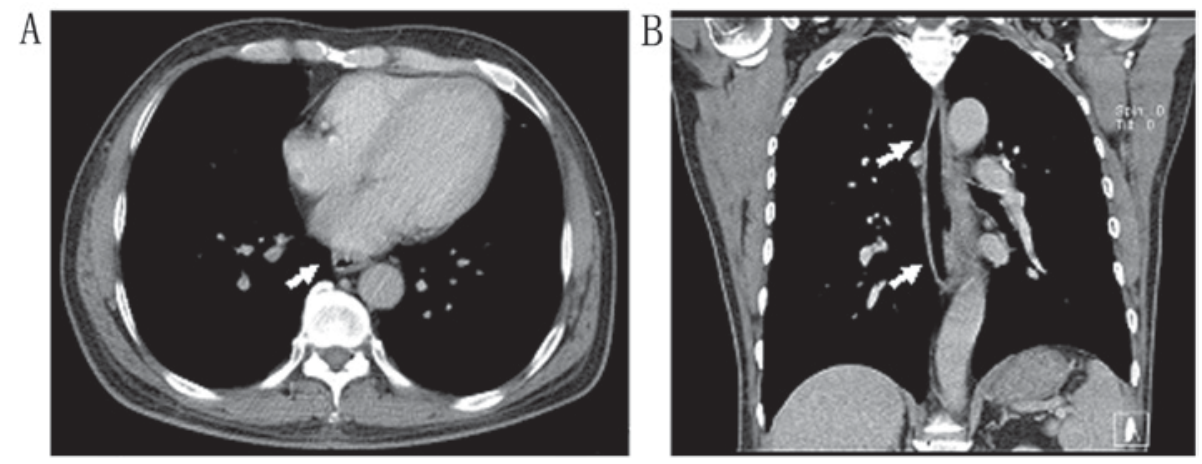

Figure 4. Chest CT scans of a patient 1 month after chemotherapy with a cisplatine/etoposide VP-16/ifosfamide/dexamethasone/mesna therapy regimen, demonstrating a marked decrease in tumor size (arrows). (A) Parenchymal phase of contrast-enhanced CT scan image (axial); and (B) parenchymal phase of contrast-enhanced CT scan image (coronal). CT, computerized tomography.

follow-up evaluation in July 2014. At the time of writing, the patient has survived for 24 months with a good quality of life without postprandial fullness or difficulty swallowing solid food. Informed consent was obtained from the patient for the present case report.

\section{Literature review}

The present study searched Pubmed, Medline, Google Scholar, Chinese Biomedicine Database and the China Journal Full Text Database for articles published in English and Chinese. The search term used was: Lymphoma, Extranodal NK-T-Cell (MeSH). Initially, five relevant items were identified using PubMed, Medline, Google Scholar, Chinese Biomedicine
Database and China Journal Full Text Database. Publication dates ranged between June 1997 and June 2015. Each publication was reviewed and, subsequently, five original studies were selected. The characteristics of 6 patients with primary NK/T-cell lymphoma of the esophagus is presented in Table I.

\section{Discussion}

Extranodal NHL primarily occurs in the gastrointestinal tract, with between 48 and 50\% occurring in the stomach, between 30 and $37 \%$ in the intestine, and between 12 and $13 \%$ in the ileocecal region (2). The total number of patients with primary esophageal lymphoma was $<35$ cases and the least common type is diffuse large B-cell type NHL. According 
to a published study, Taal et al (10) identified only 2 cases of primary NHL out of a total of 37 cases of esophageal NHL. NK/T-cell lymphoma is a distinct subgroup of diffuse large B-cell type NHL with a diverse spectrum of morphologies (11) and accounts for between 2 and $8 \%$ of NHL in Asia, and $<2 \%$ in Europe (12). Primary esophageal NK/T cell lymphoma is rare: In a review of published studies between 1997 and June 2015 (Pubmed, Medline, Google Scholar, Chinese Biomedicine Database and the China Journal Full Text Database), only 6 cases were identified (4-8). Between May 2010 and July 2014, 346 patients with extranodal NHL confirmed by surgery and pathology were retrospectively reviewed, and only 2 cases were esophageal ( $0.6 \%)$; an indication that the lymphoid tissue present in a particular segment has an association with the incidence of lymphoma in the gastrointestinal tract (13).

Esophageal NK/T-cell lymphoma typically arises in adult patients aged between 40 and 61 years, with the mean age of 49 years (almost $50 \%$ of described cases occurred in patients over the age of 49). Esophageal NK/T-cell lymphoma is primarily in male patients, with a male to female ratio of 5:1 (4-8). Patients with esophageal lymphoma may present with dysphagia (3), odynophagia (14) and weight loss (9). Additional clinical features include fever, night sweats (15), pharyngeal pain (16) and epigastric pain (17), and generalized weakness and dizziness (18) may also be present. Dysphagia symptoms are common due to the increased thickening of the esophageal wall. However, a patient with esophageal NHL without any clinical symptoms has been previously reported (19). Previous studies have demonstrated that Epstein-Barr virus (EBV) infection has been determined in virtually all patients with gastrointestinal NK/T cell lymphoma $(20,21)$. Although the association between EBV and esophageal NK/T cell lymphoma is well-understood through previous clinical studies $(5,22)$, it is typically controversial. Kim et al (20) identified that the frequency of EBV-positivity is decreased in the esophagus due to the limited number of EBV RNA tests available and the inclusion of $\mathrm{CD} 3 \varepsilon^{+} \mathrm{CD} 56^{-}$EBV. However, Lee et al (5) revealed that viral esophagitis may serve a role in the origin and development of NK/T cell lymphoma, and noted benign conditions that mimic viral esophagitis.

The criteria for primary extranodal lymphoma include the following: i) Superficial lymph node involvement is absent; ii) the initial symptoms are confined to the primary site; iii) the tumor is limited to the primary region with an absence of adjacent lymphoma or lymphoblastic leukemia; and iv) the possibility of secondary lymphomas is excluded by test results. In the present study, the patient presented to hospital with a 2-year history of postprandial fullness and difficulty swallowing solid food. The immunohistochemical staining suggested that the tumor meets the diagnostic criteria for NK/T-cell lymphoma. Although biopsies, obtained through esophagogastroduodenoscopy, are useful when making a definitive diagnosis of esophageal NK/T-cell lymphoma, misdiagnosis is often observed. This diagnostic delay is due to the character of vascular invasion, which is a strong factor of inflammatory cell infiltration, and the result of large-scale necrosis is the main cause of misdiagnosing esophageal NK/T cell lymphoma. Esophageal NK/T cell lymphoma is diagnosed by immunophenotyping $(5,6,11,12)$. The typical phenotype is the expression of NK cell markers (including CD56), T cell-associated antigens 
(including CD3 and CD2) and expression of cytotoxic markers (including TIA-1, perforin and granzyme B), with negative results for CD20 and CD23 $(5,6,11,12)$. The present case demonstrated the immune characteristics of tumor cells are typical of NK/T cell lymphoma: $\mathrm{CD}^{+}, \mathrm{CD}^{+} 6^{+}, \mathrm{TIA}^{-} 1^{+}$and granzyme $\mathrm{B}^{+}$. Radiographic characteristics of esophageal lymphoma have revealed a variety of abnormalities which are similar to lymphoma in other gastrointestinal locations (10). Additionally, Crook and Robinson (23) identified negative biopsy results in a patient with a history of esophageal large B-cell lymphoma, which suggests that analysis of cytological specimens may be required to diagnose lymphoma. According to a previous study which analyzed clinical and pathological results (9), primary esophageal lymphomas typically arise in the lower esophagus. In the present study, the esophageal wall, extending from the upper to the lower portion, was extensively thickened. A previous study (24) suggested that ulceration, or tumors which are ulceroinfiltrative, was a feature of esophageal NK/T cell lymphomas (24). However, owing to the limited number of distinguishing symptoms, including ulcerations from other types of esophageal inflammation, it was not possible to achieve a definitive diagnosis from the esophagogastroduodenoscopy (24).

The clinical diagnosis of esophageal NK/T-cell lymphoma is rare since it is difficult to make a correct differential diagnosis on the basis of esophageal symptoms in patients presenting with a fungal or viral infection, therapy-related mucositis or reflux esophagitis (25). The diagnosis of esophageal NK/T-cell lymphoma is often overlooked as it can present as dysphagia, odynophagia or pharyngeal pain. Since esophageal NK/T-cell lymphoma presents with the lack of specific clinical symptoms and signs, the early diagnosis and appropriate treatment of this lymphoma may be delayed. Patients with human immunodeficiency virus infection and acquired immune deficiency syndrome may have an increased risk of developing esophageal lymphoma; however, this is rare. Immunohistochemical analysis of esophageal node and intestinal polyp biopsies may assist in identifying patients with esophageal NK/T-cell lymphoma. Since esophageal $\mathrm{NK} / \mathrm{T}$-cell lymphoma is a relatively rare type of tumor arising from the esophageal submucosa, the primary reasons for misdiagnosis are inadequate analysis and improper biopsy. Examination by brush cytology may assist in diagnosing this type of tumor.

Despite the diagnosis of esophageal NK/T-cell lymphoma being clear, the disease was incurable (11). Primary gastrointestinal lymphoma is an aggressive malignancy with vascular destruction, tissue necrosis and a poor prognosis, with overall 5-year and 5-year disease-free survival rates of 47 and 40\%, respectively (11). A total of $79 \%$ of patients with primary gastrointestinal lymphoma succumbed within 1 year of diagnosis (11). It has been established that prognostic factors include stage of the disease, extent of surgical resection, response to treatment, serosal involvement, multimodality treatment, and performance status of the patient (11). Therefore, clinically suspected malignant lymphoma requires early diagnosis and treatment for the best outcome for the patient; however, an effective standard treatment for this type of tumor remains unknown. Fujihara et al (6) and Zhang et al (4) have suggested that systemic chemotherapy combined with concurrent radiotherapy may be an effective treatment for esophageal NK/T-cell lymphoma. Lee et al (5) identified a 45-year-old male with esophageal NK/T-cell lymphoma who was treated by salvage SMILE chemotherapy (dexamethasone, methotrexate, ifosfamide, L-asparaginase and etoposide). Primary NK/T cell lymphoma of the esophagus is rare; however, pathological and immunohistochemical examinations of biopsy specimens may be useful in making an early diagnosis.

\section{References}

1. Wu X, Li P, Zhao J, Yang X, Wang F, Yang YQ, Fang F, Xu Y, Zhang $\mathrm{H}$, Wang $\mathrm{WY}$ and Yi C: A clinical study of 115 patients with extranodal natural killer/T-cell lymphoma, nasal type. Clin Oncol (R Coll Radiol) 20: 619-625, 2008.

2. Ghimire P, Wu GY and Zhu L: Primary esophageal lymphoma in immunocompetent patients: Two case reports and literature review. World J Radiol 2: 334-338, 2010.

3. Mori T, Komeno T and Ohtani H: Mantle cell lymphoma-like solitary polypoid tumor of the esophagus: A case report. Cases J 2: 6646, 2009.

4. Zhang S, Wang H and Leng DN: Clinicopathological observation on 2 cases of nasal-type NK/T-cell lymphoma in the esophagus. Zhonghua Zhong Liu Za Zhi 30: 767-769, 2008 (In Chinese).

5. Lee SR, Park EK, Won NH and Kim BS: Esophageal involvement by extranodal natural killer $\mathrm{T}$ cell lymphoma, nasal type, mimicking Epstein Barr viral esophagitis in a tonsillar lymphoma patient undergoing chemoradiation therapy. Asia Pac J Clin Oncol 6: 149-154, 2010.

6. Fujihara S, Mori H, Kobara H, Nishiyama N, Kobayashi M and Masaki T: Esophageal natural killer (NK)/T cell lymphoma of true natural killer cell origin. Endoscopy 46 (Suppl 1 UCTN): E77-E78, 2014.

7. Song Y, Huang Y, Hu Y and He C: Primary NK/T cell lymphoma of the esophagus: A case report. Chin J Oncol: 400, 2014 (In Chinese).

8. Tang J and Li D: Primary extranodal NK/T cell lymphoma, nasal type of the esophagus-gastric cardia: A case report. Chin J Diffic Compl Cas: 645-66, 2014 (In Chinese).

9. Hosaka S, Nakamura N, Akamatsu T, Fujisawa T, Ogiwara Y, Kiyosawa K, Hidaka E, Ota H, Katsuyama T and Inagaki H: A case of primary low grade mucosa associated lymphoid tissue (MALT) lymphoma of the oesophagus. Gut 51: 281-284, 2002.

10. Taal BG, Van Heerde P and Somers R: Isolated primary oesophageal involvement by lymphoma: A rare cause of dysphagia: Two case histories and a review of other published data. Gut 34: 994-998, 1993.

11. Li JZ, Tao J, Ruan DY, Yang YD, Zhan YS, Wang X, Chen Y, Kuang SC, Shao CK and Wu B: Primary duodenal NK/T-cell lymphoma with massive bleeding: A case report. World J Clin Oncol 3: 92-97, 2012.

12. Zheng S, Ouyang Q, Li G, Xu H, Jiang M, Cui D, Xue L and Li J: Primary intestinal NK/T cell lymphoma: A clinicopathologic study of 25 Chinese cases. Arch Iran Med 15: 36-42, 2012.

13. Bandyopadhyay SK, Moulick A and Dutta A: Primary duodenal lymphoma producing obstructive jaundice. J Assoc Physicians India 55: 76-77, 2007.

14. Coppens E, El Nakadi I, Nagy N and Zalcman M: Primary Hodgkin's lymphoma of the esophagus. AJR Am J Roentgenol 180: 1335-1337, 2003.

15. Radin DR: Primary esophageal lymphoma in AIDS. Abdom Imaging 18: 223-224, 1993.

16. Doki T, Hamada S, Murayama H, Suenaga H and Sannohe Y: Primary malignant lymphoma of the esophagus. A case report. Endoscopy 16: 189-192, 1984.

17. Kalogeropoulos IV, Chalazonitis AN, Tsolaki S, Laspas F, Ptohis N, Neofytou I and Rontogianni D: A case of primary isolated non-Hodgkin's lymphoma of the esophagus in an immunocompetent patient. World J Gastroenterol 15: 1901-1903, 2009.

18. Lin CC, Lai HW, Tsai MC, Chen TH, Tseng SW, Chang H and Chen TY: Extranodal marginal zone B-cell lymphoma of the gastrointestinal tract sparing only the esophagus: A case report. Turk J Gastroenterol 23: 58-62, 2012.

19. Kurihara K, Fukui A and Kumano S: Malignant lymphoma of the esophagus associated with macroglobulinemia: Report of a case. Pathol Int 44: 712-715, 1994. 
20. Kim TM, Lee SY, Jeon YK, Ryoo BY, Cho GJ, Hong YS, Kim HJ, Kim SY, Kim CS, Kim S et al: Clinical heterogeneity of extranodal NK/T-cell lymphoma, nasal type: A national survey of the Korean cancer study group. Ann Oncol 19: 1477-1484, 2008.

21. Bautista-Quach MA, Ake CD, Chen $M$ and Wang J: Gastrointestinal lymphomas: Morphology, immunophenotype and molecular features. J Gastrointest Oncol 3: 209-225, 2012.

22. Jaffe ES, Chan JK, Su IJ, Frizzera G, Mori S, Feller AC and Ho FC: Report of the workshop on nasal and related extranodal angiocentric T/Natural killer cell lymphomas. definitions, differential diagnosis and epidemiology. Am J Surg Pathol 20: 103-111, 1996.
23. Crook TW and Robinson RA: Dysphagia in a patient with a history of large B-cell lymphoma: Esophageal disease with negative biopsy findings. Diagn Cytopathol 26: 167-169, 2002.

24. Kim JH, Lee JH, Lee J, Oh SO, Chang DK, Rhee PL, Kim JJ, Rhee JC, Lee J, Kim WS and Ko YH: Primary NK-/T-cell lymphoma of the gastrointestinal tract: Clinical characteristics and endoscopic findings. Endoscopy 39: 156-160, 2007.

25. Santra G: Oesophageal involvement in mantle cell lymphoma. Singapore Med J 51: e201-e203, 2010. 